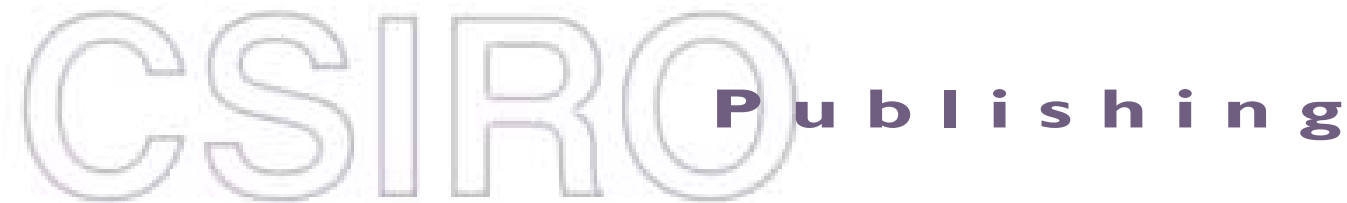

\section{Publications of the Astronomical Society of Australia}

Volume 19, 2002

(C) Astronomical Society of Australia 2002

An international journal of astronomy and astrophysics

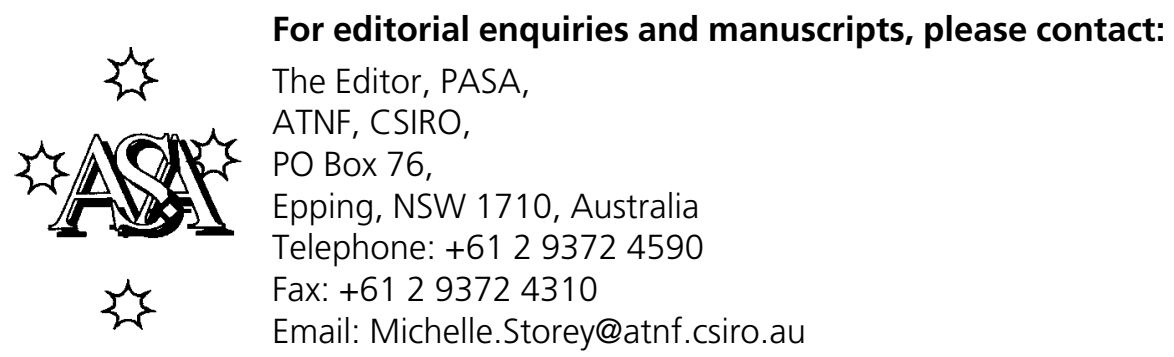

For general enquiries and subscriptions, please contact: CSIRO Publishing PO Box 1139 (150 Oxford St)

Collingwood, Vic. 3066, Australia

Telephone: +6139662 7666

Fax: +61 396627555

Email: publishing.pasa@csiro.au

C S I RO

PUBLISHING Published by CSIRO Publishing

for the Astronomical Society of Australia

www.publish.csiro.au/journals/pasa 


\title{
Spectral Variability of BL Lac Objects
}

\author{
R. D’Amicis ${ }^{1}$, R. Nesci ${ }^{1}$, E. Massaro ${ }^{1}$, M. Maesano ${ }^{1}$, \\ F. Montagni ${ }^{1}$ and F. D'Alessio ${ }^{2}$ \\ ${ }^{1}$ Department of Physics, University La Sapienza, P.le Aldo Moro 2, I-00185 Roma, Italy \\ Roberto.Nesci@uniroma1.it \\ ${ }^{2}$ Osservatorio Astronomico di Roma, via di Frascati 33, I-00040 Monteporzio Catone (Roma), Italy \\ dalessio@coma.mporzio.astro.it \\ Received 2001 July 25, accepted 2002 March 14
}

\begin{abstract}
We present the relation between optical luminosity and spectral slope for eight BL Lac objects (3C66A, PKS 0422+00, S5 0716+71, OJ 287, ON 231, OQ 530, S5 1803+78 and BL Lacertae), derived from B, V, R, and I observations spanning a time interval of about 5 years. Four objects show a marked correlation between spectral slope and luminosity, being bluer when brighter, while for the other four the correlation is weaker or absent. Possible explanations are briefly discussed in the framework of current models of the BL Lac phenomenon.
\end{abstract}

Keywords: BL Lacertae objects: individual (3C 66A, PKS 0422+004, S5 0716+71, OJ 287, ON 231, OQ 530, S5 1803+78, BL Lac)

\section{Introduction}

BL Lacertae objects are the most variable extragalactic objects, with emission extending from the radio to X-rays, and sometimes to gamma rays. Their emission is generally believed to come from a beamed relativistic jet, nearly aimed in the observer's direction. The beaming factor $\delta$ has been estimated for a number of objects, ranging from 0.1 to 10 or more (Ghisellini et al. 1993; Lähteenmäki \& Valtaoja 1999).

In the plane $\log \left(v F_{v}\right)$ vs $\log (v)$, the spectral energy distribution (SED) is roughly bell-shaped: according to the position of the SED peak, BL Lac objects were classified as (Padovani \& Giommi 1995) low energy peaked (LBL) or high energy peaked (HBL). In the first class the peak is located in the infrared $\left(\sim 10^{13} \mathrm{~Hz}\right)$ while in the second one it is located in the UV or X-rays. Further studies showed the existence of intermediate objects, i.e. with the SED peak at optical frequencies, therefore called IBL (Giommi et al. 1999).

For radio selected objects, like those studied in this work (mostly LBL), the optical spectrum is the high energy tail of the synchrotron emission of relativistic electrons, and can be fitted with a power law $\left(\mathrm{F}(v)=\mathrm{A} v^{\alpha}\right)$ with typical slopes $\alpha$ between -1 and -2 (see e.g. Moles et al. 1985).

A correlation between the spectral slope and the source intensity has been searched for since the early times of BL Lac research (e.g. Gear et al. 1986) and could be easily explained if the luminosity increase was due to the injection of fresh electrons with an energy distribution harder than that of the previous, partially cooled, ones (see e.g. Kirk, Rieger, \& Mastichiadis 1998; Mastichiadis \& Kirk 2002 , for recent models). In this paper we present a search for spectral variability in the optical B, V, R, I bands for 8 radio selected BL Lac objects.

\section{Observations and Results}

The observations were made in the years 1995-99 mostly with the $50 \mathrm{~cm} \mathrm{f} / 4.5$ Newtonian reflector at Vallinfreda (Roma) with a front-illuminated CCD camera and B, V, $\mathrm{R}$, I Johnson-Cousins filters. Typical exposure times were 5 minutes, providing internal errors from 0.01 to $0.03 \mathrm{mag}$. A minor number of observations were made with the $70 \mathrm{~cm}$ f/8 Ritchey-Chretien telescope at Monte Porzio (Roma) and the $32 \mathrm{~cm} \mathrm{f} / 4.5$ Newtonian telescope at Greve in Chianti (Firenze), both using identical back-illuminated CCD cameras. Given the fast variability of BL Lac objects, we used nearly simultaneous (taken within $20 \mathrm{~min}$ ) B, V, $\mathrm{R}$, I images only for this paper.

Differential photometry with respect to 3 or 4 nearby comparison stars was performed with IRAF/apphot using a $5^{\prime \prime}$ radius aperture. Reddening corrections for the foreground Galactic absorption were made according to Schlegel, Finkbeiner, \& Davis (1998). Conversion from apparent magnitudes to flux densities were made assuming the zero magnitude equivalent flux density given by Mead et al. (1990, their Table I, page 189).

Spectral slopes were derived using a least squares fitting with a straight line in the $\log \left(\mathrm{F}_{v}\right)$ vs $\log (v)$ plane. In Table 1 we report the principal results of our research. Column 1 gives the object name, column 2 the number of 4-band photometric points used, column 3 the range of variability of the spectral slope $\alpha$ (max-min), column 4 the average $\alpha$ value, column 5 the variability range in magnitudes, column 6 the average $\mathrm{R}$ magnitude, column 7 the linear correlation coefficient between $\mathrm{R}$ and $\alpha$, column 8 the adopted colour excess, column 9 the redshift $z$, and column $10 \delta$ according to Lähteenmäki \& Valtaoja (1999). Objects are listed in order of correlation strength. The plot of $\alpha$ vs R for each object is given in Figure 1. 
Table 1. Observational data

\begin{tabular}{lrccccccrc}
\hline Source & Data & $\alpha_{\text {range }}$ & $\langle\alpha\rangle$ & $\mathrm{R}_{\text {range }}$ & $\langle\mathrm{R}\rangle$ & $\mathrm{r}$ & $\mathrm{E}(\mathrm{B}-\mathrm{V})$ & $z$ & $\delta$ \\
\hline 3C 66A & 55 & 0.81 & -1.21 & 2.06 & 14.20 & 0.785 & 0.084 & 0.444 & 2.0 \\
BL Lac & 24 & 0.70 & -1.80 & 1.99 & 14.01 & 0.779 & 0.329 & 0.069 & 3.9 \\
ON 231 & 98 & 0.77 & -1.29 & 2.02 & 13.69 & 0.767 & 0.023 & 0.102 & 1.6 \\
OQ 530 & 45 & 0.72 & -1.56 & 1.31 & 15.00 & 0.737 & 0.013 & 0.152 & 2.7 \\
PKS 0422 & 39 & 0.56 & -1.18 & 2.07 & 14.12 & 0.529 & 0.101 & - & 1.7 \\
S5 1803 & 77 & 0.53 & -1.62 & 2.15 & 15.12 & 0.423 & 0.052 & 0.680 & 6.4 \\
S5 0716 & 114 & 0.74 & -1.40 & 2.15 & 13.66 & 0.367 & 0.031 & - & - \\
OJ 287 & 41 & 0.77 & -1.59 & 0.96 & 15.33 & 0.344 & 0.028 & 0.306 & 18.0 \\
\hline
\end{tabular}

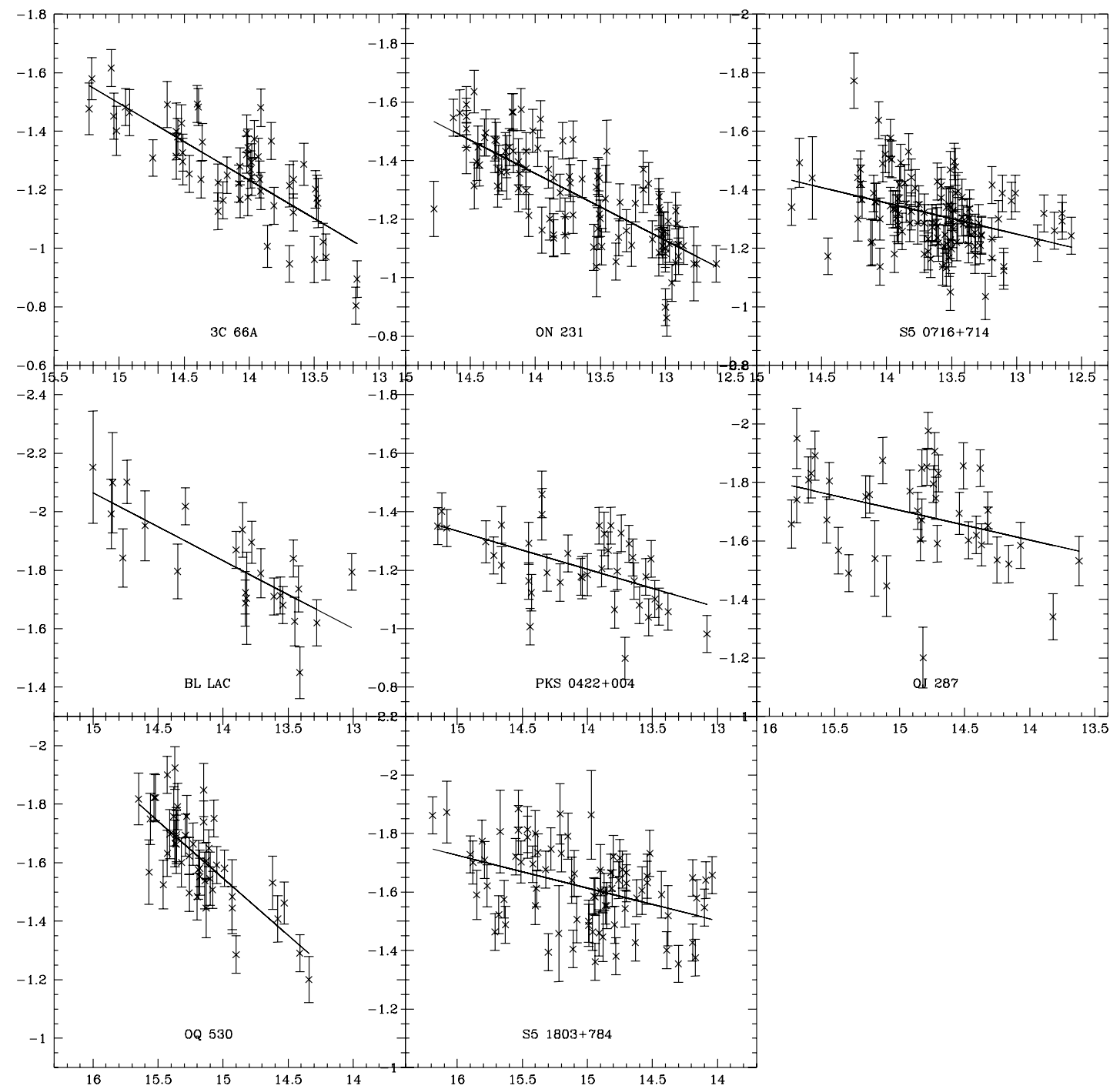

Figure 1 Spectral index $\alpha$ vs R mag for our BL Lac objects. The formal best fitting line is indicated for each source. For ease of comparison all figures have the same range in magnitude (2.7) and in spectral slope (1.2).

The main results of our monitoring may be summarised as follows:

i) We found that the spectral slope of our BL Lacs does vary significantly in the optical band. The general sense of this variation is that the spectrum becomes bluer when the source is brighter, as already observed by several authors for a few BL Lac objects (e.g.
Ghisellini et al. 1997; Maesano et al. 1997; Massaro et al. 1996; Tosti et al. 1998; Takalo et al. 1996; but see de Diego et al. 1997 for an opposite case).

ii) The correlation is not equally good for all our objects: only 4 out of our sample of 8 show a correlation $r>0.7$, apparently those with an estimated lower value of beaming. 
iii) The observed ranges of variability of $\alpha$ and flux density are rather similar for all 8 objects, despite their large spread in absolute luminosity and $z$.

iv) There is no correlation between the average $\alpha$ and the average absolute luminosity, nor between average $\alpha$ and $z$.

\section{Discussion}

In the framework of current synchrotron emission models, a spectral hardening is expected to be correlated with a luminosity increase in the optical band (see e.g. Kirk et al. 1998). The effect would be more marked for sources with the emission peak at longer wavelengths.

Our first two findings, therefore, are in agreement with current synchrotron models. In particular, the ranking in correlation strength found in our monitoring might be interpreted as a ranking in the position of the emission peak. In this scenario the first 4 sources should be classified as LBL in the scheme of Padovani \& Giommi (1995), while the remaining ones should be considered intermediate ones (IBL).

An increase of $\delta$ implies an increase of the radiation frequency in the observer's reference frame: therefore, in the optical band the observer sees a spectral interval of intrinsically lower frequency, which has a flatter slope in LBL objects. If the intrinsic SED were equal, more beamed objects would show flatter spectra, which does not seem to be the case in our (somewhat small) sample. Further simultaneous multiwavelegth observations will help to clarify the interpretation.

This last correlation would be expected if the objects had all the same intrinsic spectral slope, because the synchrotron emission becomes steeper going toward high frequencies for LBL objects: given that the spectrum is more redshifted for more distant objects, the farthest ones should have apparently steeper spectra. The lack of correlation suggests that the spectral energy distributions of our sample objects are intrinsically different.

There is however the possibility, already seen in some HBL objects (see Pian 2002) and in ON 231 (Massaro et al. 1999) that the emission peak is not fixed in time for a given object, requiring therefore a more complex modelling of these sources.

\section{References}

de Diego, J. A., Kidger, M. R., Gonzales-Perez, J. N., \& Lehto, H. J. 1997, A\&A, 318, 331

Gear, W. K., et al. 1986, ApJ, 304, 295

Ghisellini, G., Padovani, P., Celotti, A., \& Maraschi, L. 1993, ApJ, 407,65

Ghisellini, G., et al. 1997, A\&A, 327, 61

Giommi P., et al. 1999, A\&A, 351, 59

Kirk, J. G., Rieger, F. M., \& Mastichiadis, A. 1998, A\&A, 333, 452

Lähteenmäki, A., \& Valtaoja, E. 1999, ApJ, 521, 493

Maesano, M., Montagni, F., Massaro, E., \& Nesci, R. 1997, A\&AS, 122,267

Massaro, E., et al. 1996, A\&A, 314, 87

Massaro, E., et al. 1999, A\&A, 342, L49

Mastichiadis, A., \& Kirk, J. G. 2002, PASA, 19, in press

Mead, A. R. G., Ballard, K. R., Brand, P. W. J. L., Hough, J. H., Brindle, C., \& Bailey, J. A. 1990, A\&AS, 83, 183

Moles, M., Garcia-Pelayo, J. M., Masegosa, J., \& Aparicio, A. 1985, ApJS, 58, 255

Padovani, P., \& Giommi, P. 1995, ApJ, 444, 567

Pian, E. 2002, PASA, 19, 49

Schlegel, D. J., Finkbeiner, D. P., \& Davis, M. 1998, ApJ, 500, 525

Takalo, L. O., et al. 1996, A\&AS, 120, 313

Tosti, G., et al. 1998, A\&AS, 130, 109 